\title{
Quando l'abuso psicologico è in agguato: la sottrazione internazionale del minore. Proposte operative
}

\author{
Mimma Tafä ${ }^{*}$, Marisa Malagoli Togliatti*
}

Il contributo tratta il fenomeno della sottrazione internazionale del minore, che si verifica nelle situazioni di separazione di coppie miste: in questi casi il conflitto della coppia esita nel "rapimento" del figlio da parte di uno dei due genitori, che torna al suo Paese di origine portando con sé il minore, in uno Stato diverso da quello della sua residenza abituale. Il contributo descrive i risultati della terza rilevazione, che rientra in una ricerca qualitativa più ampia. Il campione dell'indagine è costituito da 50 famiglie interculturali, che si sono rivolte all'Autorità Centrale, di cui sono stati analizzati i fascicoli. In tutte e tre le rilevazioni effettuate, che hanno caratterizzato la ricerca, si registra la mancanza di interventi psicologici e questo fa ipotizzare un rischio di abuso psicologico per il minore sottratto; a tal proposito vengono proposti alcuni interventi specifici per queste situazioni.

Parole chiave: sottrazione internazionale del minore, coppia mista, separazione, spazio neutro.

When psychological abuse is just around the corner: International child abduction. Operational proposals.

Our contribution deals with the phenomenon of international child abduction, which may occur when interracial and cross-cultural couples separate; in such circumstances the outcome of marital conflict may be the "abduction" of the child by a parent, who returns to his/her native Country with the child, in a State that is not their usual place of abode. Our contribution describes the results of the $3^{\text {rd }}$ survey, which is part of a broader qualitative research. The survey sample is composed of 50 cross-cultural families that filed a petition to the Central Authority. Their files were examined. The three surveys conducted (for our research) all stated a lack of psychological interventions, hence allowing us to hypothesize the risk of psychological abuse for the abducted child. According to these data, our contribution suggests some specific interventions to cope with such situations:

Keywords: international child abduction, interracial and cross-cultural couple, separation, neutral space.

\footnotetext{
* Ricercatore, Dipartimento di Psicologia Dinamica e Clinica, Facoltà di Medicina e Psicologia, Sapienza Università di Roma.

Professore Ordinario, Dipartimento di Psicologia Dinamica e Clinica, Facoltà di Medicina e Psicologia, Sapienza Università di Roma.

Indirizzare le richieste a: Mimma Tafà,mimma.tafa@uniroma1.it.

Un ringraziamento particolare alla prof.ssa L. Iesu per il prezioso contributo allo svolgimento della ricerca. Un ringraziamento anche alla dott. G. Giarola per il contributo alla stesura dell'articolo relativo alla ricerca ed analisi delle fonti bibliografiche.
}

35

Maltrattamento e abuso all'infanzia, Vol. 15, n. 2, giugno 2013, pp. 35-64 


\section{Introduzione}

Il fenomeno della sottrazione internazionale del minore (Iesu, Fiorelli, \& Tafà, 2008; Tafà, Malagoli Togliatti, \& Milazzo, 1998; Tafà, 1999; Tafà \& Iesu, 2007; Tafà, 2009) si può verificare in concomitanza della separazione tra partner di diversa provenienza culturale: in questi casi il conflitto della coppia può sfociare nel "rapimento" del figlio da parte di uno dei due genitori, che torna al proprio Paese di origine, portando con sé il minore (Re \& Sanchez, 2003). Il bambino così - per effetto della sottrazione - deve affrontare una nuova permanenza in uno Stato diverso da quello di residenza abituale. Nel timore di non ottenere la custodia esclusiva al momento della separazione, uno dei genitori generalmente quello straniero - può essere indotto, quindi, a sottrarre il figlio ed a condurlo nel proprio Paese di origine, sradicandolo così dal suo contesto relazionale, sociale e geografico.

Emerge, dunque, come il fenomeno della sottrazione rechi in sé il problema dell'identità del bambino, che implica il diritto alla conservazione delle proprie origini e delle relazioni familiari, sociali e culturali. La Teoria dell'Identità Sociale, infatti, sostiene che l'individuo tende a raggiungere un'immagine di se stesso positiva caratterizzata da due aspetti distinti: l'identità personale e l'identità sociale. Mentre la prima include attributi specifici e si riferisce a come l'individuo vede se stesso, la seconda implica quella parte del concetto di Sé che deriva dalla consapevolezza di essere membro di un gruppo sociale e si riferisce a come l'individuo vede lo stesso gruppo sociale a cui appartiene (Tajfel \& Turner, 1986). Analogamente, Minuchin (1976, p. 49) ci ricorda che: «(...) la famiglia risponde a due obiettivi. Uno è interno: la protezione psico-sociale dei suoi membri; l'altro esterno: l'accomodamento a una cultura e la propagazione di quella cultura».

Nonostante la famiglia attualmente rappresenti un oggetto di studio e d'intervento significativo sia dal punto di vista clinico sia da quello empirico in ambito nazionale ed internazionale (Carli \& Rodini, 2008; Dowling \& Gorell Barnes, 2004; Fivaz-Depeursinge \& CorbozWarnery, 1999; Gambini, 2007; Mazzoni \& Tafà, 2007; Simonelli, Bighin, \& De Palo, 2012; Walsh, 2006), la ricerca psicologica non sembra aver prestato una grande attenzione ai mutamenti socio-culturali rappresentati dalle coppie miste. Questo, nonostante esse facciano parte di configurazioni familiari attualmente in crescita nel nostro Paese, in cui si affronta il difficile tentativo di far convergere in una sola genitorialità, 
due civiltà e modi di pensare estremamente diversi: «(...) partner che provengono da diverse culture, razze e religioni importano diversi valori e aspettative nel rapporto» (Walsh, 2008, p. 408). La natura multiculturale della famiglia rispecchia dunque un fenomeno sociale attuale: la diversa visione del mondo tra padri e madri, che si riconduce alle enormi differenze esistenti nei sistemi di credenze dei diversi ambiti culturali di appartenenza, vanifica lo sviluppo di un sistema di significati condiviso nella coppia. É comprensibile che la sua mancanza possa ragionevolmente rappresentare il motivo del conflitto tra i partner, che sfocia successivamente nell'allontanamento di un genitore e nella sottrazione del figlio. Il disaccordo in queste coppie binazionali sembra, dunque, avere delle ricadute inevitabili a livello intergenerazionale, sia per la presenza del conflitto sia per la mancanza di interventi che lo trattino, che si traducono in un diverso stile di parenting a cui il bambino risulta esposto: la concezione dei legami familiari risulta, infatti, essere diversa a seconda della cultura di provenienza e tali differenze si concretizzano in consuetudini, pratiche e regole familiari che riflettono le caratteristiche della stessa cultura. Non a caso, Scabini e Cigoli (2000) affermano che lo stile di parenting è un indicatore della presenzà o dell'assenza del patto genitoriale. Sembra che l'assenza del patto genitoriale in queste coppie non venga supportato dagli interventi: nessuno ricorda loro come essere bravi genitori in queste delicate evoluzioni del rapporto di coppia.

Il fenomeno della sottrazione produce numerose implicazioni psicologiche a carico del bambino. Attualmente, infatti, ricercatori di diversi ambiti della psicologia, sostenuti da evidenze empiriche, concordano nella visione sistemica secondo cui lo studio dell'individuo, e quindi in particolare dei soggetti in età evolutiva, non possa prescindere dai contesti relazionali di appartenenza, quindi dalle caratteristiche della sua famiglia e del contesto allargato di cui essa fa parte (cfr. tra gli altri Malagoli Togliatti \& Mazzoni, 2006; Mazzoni \& Tafà, 2007; Simonelli et al., 2012). Nello specifico, la letteratura evidenzia come la relazione coparentale - e quindi non solo quella coniugale, che si interseca con essa - influenzi significativamente lo sviluppo del bambino, riferendosi con il termine "coparentale", alla modalità con cui le madri ed i padri sostengono e/o indeboliscono gli sforzi parentali dell'altro (FivazDepeursinge \& Corboz-Warnery, 1999).

In particolare, questo contributo è rivolto allo studio di quel tipo di famiglie, sempre più diffuse nel nostro Paese, ossia le famiglie separate, nelle quali si registra un calo delle capacità genitoriali in concomitanza 
dell'evento separativo. Questo momento di transizione viene segnalato con il termine di "latitanza genitoriale" per indicare la possibile distrazione parentale, rispetto ai propri compiti, nei primi due anni dopo la separazione (Hetherington, Law, \& O'Connor, 1993), che talvolta può procurare delle conseguenze evidenti a carico dei figli (Cigoli, 2006; Emery, 2008). Non si può certo trascurare come la separazione sia considerata attualmente dalla letteratura come un evento capace di mettere a rischio l'organizzazione funzionale della famiglia (Malagoli Togliatti \& Tafà, 2005; Gollini, Tafà, \& Malagoli Togliatti, 2001; Tafà, 2001a, 2001b; Tafà \& Micci, 2004). Il fenomeno della sottrazione internazionale del minore, che si può verificare talvolta in questi casi, mette in grave pericolo la continuità dell'esercizio genitoriale. Certi Autori ne suggeriscono addirittura una connessione (Douzenis, Kontoangelos, Thomadaki, Papadimitriou, \& Lykouras, 2012; Greif \& Hegar, 1994), come se la separazione delle coppie miste rendesse altamente probabile la sottrazione del bambino. Il suo verificarsi, infatti, ostacola l'aecesso del bambino ad entrambi i genitori, diversamente da quanto è segnalato attualmente ancora dalla letteratura come necessario per un suo sano sviluppo. Si tratta di un accesso non solo pratico, ossia legato alla frequentazione, ma anche di natura simbolica, poiché la sottrazione spesso implica la perdita del legame con l'altro genitore e la sua famiglia, nonché con il contesto socio-culturale. Questo avviene non solo nel quotidiano, ma anche nella vita mentale del bambino: infatti, nelle relazioni familiari sono contemporaneamente attivi i due livelli, quello delle rappresentazioni e quello dei modelli interattivi (Cigoli, 2006). Nella sottrazione, sia l'alleanza coniugale sia quella genitoriale risultano compromesse: infatti, in questi casi non si può raggiungere la cogenitorialità, che rappresenta la capacita déi genitori di sostenersi a vicenda nei ruoli familiari, nonostante la loro separazione. La cogenitorialità, certamente auspicabile in queste situazioni, insieme ad un buon funzionamento triadico, rappresenta un fattore protettivo per i figli (Malagoli Togliatti \& Lubrano Lavadera, 2008). Eventuali rotture lungo tale continuità relazionale, rappresentano, infatti, il danno maggiore alla costruzione della persona. In questi casi, i fattori di rischio per la crescita del minore sono certamente legati alla disfunzionalità della triade familiare successiva alla sottrazione, che minaccia la continuità della coordinazione a tre, ma anche ai numerosi cambiamenti connessi al suo rapimento:

- innanzitutto la situazione familiare/relazionale e, quindi, quella affettiva nonché abitativa. Il minore si allontana drasticamente da un ge- 
nitore e vive con l'altro - con il quale non può che stabilire un rapporto esclusivo - cambiando casa e, quindi, i riferimenti dei suoi spazi vitali, cambiando altresì le consuetudini di vita quotidiana che ha costruito nel suo ambiente;

- il minore è costretto ad un cambiamento ampio delle sue relazioni affettive: certamente quelle familiari, intendendosi non solo il genitore che subìsce la sottrazione, ma anche la presenza di fratelli laddove la famiglia ne considera, dei nonni e di tutte le figure affettive della parentela. Non è trascurabile la portata intergenerazionale della separazione, in quanto coinvolge anche le famiglie d'origine dei partner e della comunità più allargata. Entrambi in questo caso sono portatori di diversità culturali e religiose, che la coppia mista non è riuscita ad integrare al suo interno. Può accadere, pertanto, che i nonni possano stimolare le mosse conflittuali dei propri figli, scatenando una faida ideologica e religiosa, che il bambino conteso "paga" a caro prezzo. Dunque, la perdita di molteplici legami familiari per il bambino, ma anche quella con i pari di eta della scuola di appartenenza;

- in generale, il minore è costretto ad un cambiamento dell'ambiente di vita storico-geografico che partecipa significativamente alla struttura identitaria del bambino o dell'adolescente (Cigoli, 2006);

- così, il figlio viene ad appartenere ad un solo genere e ad un'unica stirpe: il genere, quello del genitore sottraente, e la stirpe, la sua famiglia d'origine. I modelli ai quali ispirarsi riguardano pertanto esclusivamente il genitore sottraente e la sua famiglia, che il minore è costretto ad adottare all'indomani della sottrazione, "dimenticando" gli altri;

- il figlio, inoltre, deve far fronte alla nuova residenza dopo la sottrazione, che avyia nuove forme di relazione: può essere solo con il genitore sottraente, con lui e la sua famiglia d'origine o anche solo con la sua famiglia ${ }^{1}$, ancora, con lui ed il nuovo partner. In ogni caso la sottrazione prevede una convivenza con altre persone talvolta sconosciute fino ad allora, in spazi diversi da quelli finora sperimentati, spesso senza sufficienti informazioni per "annaspare" nella nuova situazione familiare.

\footnotetext{
${ }^{1}$ Quando il bambino viene lasciato dai nonni ed il genitore sottraente si allontana, è ragionevole supporre che il genitore non lo faccia tanto per consolidare il suo rapporto con il figlio, quanto per la garanzia che venga cresciuto secondo i principi culturali della propria etnia.
} 
Tutto questo con la premessa iniziale dell'allontanamento dal genitore che subisce la sottrazione e con il quale il bambino ha vissuto fino ad allora.

Non è trascurabile come questi minori, sin dall'inizio, siano alle prese con un compito difficile, ossia fare i conti con una doppia cultura di appartenenza: quella di un genitore proveniente dal Paese straniero e quella dell'altro, del Paese ospitante; già precocemente, quindi, essi sperimentano due mondi, risultano "divisi" tra due culture, tra due appartenenze. È ragionevole ipotizzare che in questi casi la separazione rappresenti l'esito di un fallimento genitoriale nel processo di integrazione delle due culture e la sottrazione del minore ne rappresenti la diretta conseguenza. I bambini diventano così ostaggio della mancata elaborazione dei due mondi culturali dei rispettivi genitori e sono costretti ad assumersi una responsabilità troppo grande per la loro età: «questo li espone al conflitto tra le due culture e alla difficoltà nel passaggio da un mondo all'altro» (Mirazita, 2008; p. 42).

Sottrazione dall'altro genitore, dunque, ma anche dai propri fratelli, dai nonni - e quindi da una parte della propria storia, a cui il minore non ha più accesso - e dal suo ambiente di vita in generale. Viene compromesso il suo processo di socializzazione, non solo per quello che riguarda il genitore, ma anche per le altre figure che ruotano attorno al bambino, quali ad esempio i fratelli, i nonni e gli amici dei genitori. In particolare, Cigoli (2006) descrive il "rischio endogamico", che consiste nella convinzione presuntuosa del genitore di pensare che una sola stirpe sia sufficiente al bambino per la costruzione della sua personalità futura, escludendo così la famiglia e la stirpe dell'ex coniuge.

La sottrazione illecita dei minori da parte di uno dei genitori è divenuta certamente piu difficile a seguito dell'applicazione della Convenzione dell'Aja del 25 ottobre 1980, ratificata in Italia nel 1995, che stabilisce il rimpatrio immediato dei minori nel proprio Paese d'origine. Nonostante l'interesse legislativo (in ambito nazionale si vedano tra gli altri: Gregori, 1998; Pazè, 2008), il fenomeno non registra attualmente un'adeguata visibilità nella riflessione psicologica dello studio delle famiglie separate. Esso non gode della giusta attenzione da parte di chi si occupa di riflettere sui danni relazionali precoci; questo, pur recando evidenti implicazioni psicologiche a carico dei protagonisti e soprattutto del minore coinvolto. Nell'ambito psicologico risulta, infatti, carente la riflessione su una modalità di intervento specifica in queste situazioni, mentre sarebbe quantomeno opportuno, se non necessario, colmare tale 
lacuna e dirigere l'interesse degli esperti del settore affinché possano riflettere adeguatamente sulle vicende relazionali che attraversano il fenomeno per mettere a punto un'adeguata risposta. Tuttavia, il fenomeno diventa oggetto di attenzione da parte delle cronache, solo in situazioni familiari eclatanti. Per questo, se da una parte lo scopo del contributo è quello di diffondere i risultati dell'indagine, dall'altra si intende sensibilizzare anche i clinici, che dovrebbero volgere lo sguardo a questo fenomeno ed ai protagonisti implicati: certamente i genitori, ma soprattutto i figli sottratti. Al contrario, dalle rilevazioni effettuate in tre momenti diversi, è emerso come in nessun caso i membri familiari protagonisti della sottrazione internazionale beneficino di un sostegno psicologico e soprattutto il minore si trova sprovvisto di un supporto al di sopra delle parti, che tuteli la sua crescita e lo protegga dal conflitto genitoriale: infatti, nel processo innescato dalla sottrazione, non emerge nessun intervento psicologico nell'immediato né successivo. D'altra parte, per le famiglie separate diffuse sul territorio nazionale è previsto un intervento specifico di Mediazione Familiare, al fine di dirimere la conflittualità tra i partner nell'interesse del bambino (Malagoli Togliatti \& Tafà, 2005). Tale intervento, pur potendo essere efficace per dirimere il conflitto anche nelle coppie miste residenti in Italia, non viene utilizzato, verosimilmente per la mancanza di una sua specificità per tali unioni. Emerge, quindi, la contraddizione di un'attenzione esclusiva alla separazione ed alle modalità di intervento possibili, in grado di supportare i protagonisti nel caso di separazione nazionale, che solitamente non è contrassegnata dalla sottrazione.

Questa "distrazione" da parte degli esperti, renderebbe il rischio per il minore anche maggiore, visto che con la sottrazione può registrare la "perdita" vera e propria di un genitore e di una parte della propria storia. A tal proposito, il contributo intende colmare la scarsa attenzione alla separazione nelle coppie miste, sempre più in aumento nel nostro Paese secondo i dati Istat ${ }^{2}$. La ragione di tale incremento, secondo alcuni autori (Gozzoli \& Regalia, 2005), è spiegabile attraverso due importanti motivazioni: per le maggiori opportunità di incontro che si sono recentemente venute a creare per persone con diverso background culturale; nonché per la maggior accettazione della società nei confronti di quelle unioni che non possono essere considerate come tradizionali. Sempre i

\footnotetext{
${ }^{2}$ Ogni anno l'Istat diffonde i principali risultati delle rilevazioni sulle separazioni e divorzi. A questo proposito si veda: www.istat.it/salastampa/comunicati/non calendario/20100721_oo/testointegrale20100 721.pdf.
} 
dati Istat indicano anche una minore durata del matrimonio in questi casi, rispetto a quello italiano (9 vs 14 anni). Ma non risulta un fenomeno circoscritto al nostro Paese: oltrepassando i confini nazionali, anche altri autori sostengono una maggiore sensibilità allo stress da parte delle coppie miste (Chan \& Wethington, 1998), riconducibile anche al contesto familiare e sociale allargato, vista la criticità della loro scelta (Usita \& Poulsen, 2003).

In particolare, un'area che sintetizza le difficoltà maggiori di tali unioni risulta essere l'acquisizione della genitorialità e, quindi, le scelte educative che ovviamente tengono conto del background culturale di ogni partner (Favaro, 1996; Gozzoli \& Regalia, 2005). I genitori della coppia mista, infatti, devono assolvere a due importanti compiti che sono da ritenere fondamentali per la crescita del bambino (Gôzzoli \& Regalia, 2005): da una parte, è necessario trasmettere ai figli i valori propri della cultura di entrambi; dall'altra, essi devono sostenere il figlio nel percorso di costruzione della propria identità. Un obiettivo primario in questo percorso è la capacità dei genitori di individuare nella coppia pratiche educative condivise. Il tema delle pratiche educative riguarda principalmente come i genitori cercano di trasmettere parte della propria cultura di riferimento al figlio, in modo tale da fornirgli una solida base su cui costruire la propria identità. Nella coppia mista le scelte educative dovrebbero essere il frutto di una doppia negoziazione che, da un lato, interessi i rapporti interni alla coppia e, dall'altro, consideri il rapporto che la coppia instaura con il contesto sociale che la circonda. Ad esempio, una delle sfide che si propone alla coppia mista riguarda la scelta della lingua: sarebbe opportuno in questi casi insegnare ai figli il bilinguismo. Emerge, infatti, come il genitore straniero, pur conoscendo ed usando appropriatamente la lingua della comunità di accoglienza, ricorra alla lingua madre per comunicare le emozioni e gli aspetti maggiormente legati alla propria identità (Burk, 2004): in questo modo sente di passare ai figli qualcosa di sé e della propria cultura. In particolare, in una ricerca condotta da Favaro (1996) è emerso che nel caso di bambini con padre straniero (soprattutto di origine araba) il monolinguismo dei figli è spesso accettato con dispiacere e senso di perdita; se invece è la madre ad essere straniera appare normale accantonare la lingua minoritaria a favore del codice dominante. Il bambino misto si trova così ad affrontare già precocemente sfide connesse alla pluriappartenenza, e quindi a dover integrare messaggi e riferimenti tra loro diversi - se non contrastanti - che i suoi genitori gli trasmettono. Egli dovrebbe elabo- 
rarli, modificarli e ricomporli, così da crearsi un'eredità familiare personalizzata e coerente con la propria situazione, grazie alla quale potersi svincolare dal passato dei suoi genitori e cominciare a costruirsene uno proprio, in cui ritrovare quelle parti che ha ritenuto giusto trattenere. Sarebbe importante, in questo senso, che il bambino abbia la possibilità di passare diverso tempo con i nonni, portatori di una serie di tradizioni e pratiche che possono permettergli di focalizzare maggiormente le sue origini ed aiutarlo nel percorso di definizione di se stesso. Ecco, quindi, un altro compito importante per il bambino misto: la cura del legame intergenerazionale, ossia riuscire a garantire la continuità della storia familiare impegnandosi a trovare modalità condivise di regolazione dei rapporti con entrambe le famiglie dei genitori (Gozzoli \& Regalia, 2005). Tutti questi aspetti sono certamente messi in pericolo dalla sottrazione del minore, che privilegia un genitore ed una stirpe, ostacolando i figli in questo difficile compito.

Molte sono, dunque, le caratteristiche peculiari di queste famiglie, che rappresentano una configurazione relazionale attualmente in crescita nel nostro Paese, rendendo particolarmente importante l'attenzione da parte degli esperti agli esiti conflittuali delle coppie miste che le costituiscono, visto che attualmente non gode della giusta attenzione da parte della clinica e della ricerca. Di conseguenza, il fenomeno della sottrazione internazionale del minore, ad essa connesso, e che questo contributo vuole evidenziare, appare poco conosciuto e gode di uno scarso intervento psicologico da parte degli esperti. Dall'esame della letteratura, ci si aspetta pertanto una minore attenzione alle dinamiche psicologiche dei protagonisti rispetto a indicazioni normative o sociologiche (cfr. AAVV, 2012; Edleson, Lindhorst, Mehrotra, Veneski, Lopez, \& Shetty, 2010; Lesh, 2011; Veneski, Lindhorst, \& Edelson, 2011).

L'obiettivo dell' articolo è pertanto quello di contribuire ad aumentare la visibilità del fenomeno, che risulta scarsa nella letteratura scientifica psicologica, soprattutto da un punto di vista empirico. A tal proposito, verranno utilizzati i risultati ottenuti da una rilevazione effettuata presso l'Autorità Centrale, che in Italia si trova incardinata nel Dipartimento per la Giustizia Minorile. 


\section{Indagine}

\subsection{Strumento}

Questo contributo esplorativo fa parte di un lavoro di ricerca più ampio, che ha preso l'avvio da un'iniziale raccolta dati effettuata presso il Servizio Sociale Internazionale di Roma (SSI) prima della ratifica italiana della Convenzione dell'Aja (Tafà, 1999; Tafà et al., 1998) e che attualmente regolamenta la sottrazione dal punto di vista normativo. Un'evoluzione della ricerca ha previsto una seconda rilevazione rispetto allo stesso fenomeno, sempre presso lo stesso contesto di somministrazione costituito dal SSI, ma - in questo caso - dopo la ratifica della Convenzione, per verificare l'influenza dell'applicazione della normativa sulle informazioni ottenute, mettendo a confronto le due rilevazioni (si veda: Iesu, Fiorelli, \& Tafà, 2008; Tafà, 2009; Tafà \& Iesu, 2007). La stessa Convenzione, tra le altre cose, prevede opportunamente la creazione di un Ufficio Speciale, denominato Autorità Centrale, in ogni Stato che aderisce al dettato normativo, incaricato specificamente di adempiere ad una serie di obblighi previsti dalla stessa: «l'Autorità Centrale deve assicurare l'immediato rientro dei minori e conseguire gli altri obiettivi della Convenzione» (Capo II, art. 7). A tal fine, in questo lavoro si vuole indagare attraverso questa terza rilevazione dell'indagine lo stesso fenomeno della sottrazione internazionale del minore presso l'Autorità Centrale, come già detto, deputata specificamente dalla Convenzione dell'Aja ad intervenire in queste situazioni, che in Italia si trova incardinata nel Dipartimento per la Giustizia Minorile ${ }^{3}$. Si vuole, inoltre, confrontare i risultati ottenuti con le precedenti rilevazioni.

\footnotetext{
${ }^{3}$ Le raccolte dati effettuate presso il SSI e l'Autorità Centrale del Ministero di Grazia e Giustizia, fanno parte di Progetti di Ricerca finanziati dall'Ateneo Federato della Scienza e della Tecnologia (AST) negli anni 2007 e 2008 alla Prof. ssa M. Tafà in qualità di Proponente e Responsabile. I risultati che verranno descritti, pur riconducendosi all'anno 2002, non si discostano sostanzialmente da quelli attuali, aggiornati fino all'anno 2012, per i quali il lettore può consultare il sito del Ministero di Grazia e Giustizia (www.giustiziaminorile.it). Per questo motivo, le Autrici hanno utilizzato questa somministrazione. Inoltre, un contatto informale con le operatrici dell'Autorità Centrale ha confermato ulteriormente il dato relativo alla mancanza degli interventi psicologici, per cui sono solamente "i genitori a farsene carico" se lo ritengono opportuno, perché non sono previsti dalla Convenzione, quindi, contro la 44
}

Maltrattamento e abuso all'infanzia, Vol. 15, n. 2, giugno 2013, pp. 35-64 
A questo proposito, sono stati analizzati 50 casi di sottrazione pervenuti all'Autorità Centrale Italiana nell'anno 2002, con uno strumento analogo a quello utilizzato nelle precedenti rilevazioni (Tafà, 2009; Tafà et al., 1998; Tafà \& Iesu, 2007), che è stato opportunamente ampliato, prevedendo categorie di tipo psicologico-giuridico, capaci di codificare le informazioni presenti negli archivi dell'Autorità Centrale. Il lavoro che verrà presentato, di tipo esplorativo, è basato su dati di statistica descrittiva ${ }^{4}$. Sono stati considerati: l'adesione alla Convenzione dell'Aja da parte dei Paesi implicati nella sottrazione, la fase del ciclo vitale attraversata dalla coppia al momento della sottrazione, informazioni sul minore (ossia sesso ed età al momento della sottrazione) e sulla procedura giuridica, il tipo di affidamento legale in atto in concomitanza della separazione, il responsabile della sottrazione, la durata del procedimento della sottrazione e l'eventuale rimpatrio del minore. I dati così raccolti sono stati successivamente inseriti nel pacchetto statistico SPSS ed elaborati in frequenze e percentuali.

\subsection{Risultati}

Verranno ora esposti i risultati principali di questa terza rilevazione e, come detto, messi a confronto con le due precedenti (Tafà, 2009; Tafà et al., 1998) $)^{5}$. Per facilitare il lettore da adesso: $1^{\circ}=$ Tafà et al., 1998; $2^{\circ}$

legge e pertanto considerati come "un'invadenza alle procedure effettuate dall'altro Stato".

${ }^{4}$ Lungi dall'avere pretese statistiche, il lavoro - come già detto - si propone di aumentare la conoscenza del fenomeno nella comunità scientifica. Sulla base dei risultati della ricerca esplorativa verranno formulate delle ipotesi nelle conclusioni del lavoro. Nell'indagine presentata si sono voluti confrontare i risultati ottenuti in due contesti di somministrazione diversi, a cavallo dell'applicazione italiana della Convenzione relativamente allo stesso fenomeno. La metodologia utilizzata nelle indagini precedenti è analoga a quella presentata in questo lavoro. Le autrici prevedono la continuazione dell'osservazione del trend.

${ }^{5} \mathrm{Si}$ fa presente al lettore che le informazioni ipotizzate dai Ricercatori nella fase iniziale di pre-ricerca, considerate necessarie alla esaustiva descrizione del fenomeno, si sono rivelate solo in parte presenti in questi dati provenienti dall'Autorità Centrale. In questa rilevazione sono state ottenute meno informazioni rispetto alle precedenti: il lettore interessato può, infatti, trovare nelle altre rilevazioni (Tafà et al., 1998; Tafà, 2009) maggiori categorie relative alla presenza di queste nei fascicoli esaminati. Il motivo è che in questo caso, l'accesso alle informazioni non è fornito dai fascicoli, ma dalle informazioni desunte da questi ed organizzate statisticamente dalla stessa Autorità. Queste statistiche sono state rese disponibili per l'indagine. Per questo motivo non è 
$=$ Tafà, $2009 ; 3^{\circ}=$ questo stesso articolo, vale a dire l'indagine presentata in questo contributo.

Per quanto riguarda l'adesione alla Convenzione dell'Aja nel campione dell'indagine, 1 ' $82 \%$ dei Paesi che sono entrati in contatto con l'Ufficio dell'Autorità Centrale Italiana, ha aderito alla Convenzione stessa, dato in aumento rispetto alla precedente rilevazione $\left(2^{\circ}\right)$, in cui se ne contava il $74.2 \%{ }^{6}$. Ovviamente questo dato, manca nella prima rilevazione, poiché è stata effettuata quando ancora la Convenzione non era attiva $\left(1^{\circ}\right)$.

Analogamente a quello che è emerso nelle due precedenti rilevazioni, la fase del ciclo vitale in cui avviene più frequentemente la sottrazione risulta essere, in particolare, la famiglia con bambini piccoli, in età prescolare $\left(1^{\circ}=63 \%, 2^{\circ}=54.8 \%, 3^{\circ}=48 \%\right)$, ovvero quando ancora $\mathrm{i}$ bambini non hanno fatto il loro ingresso nella scuola elementare, fase che coincide con l'inizio della loro autonomizzazione e della conoscenza del mondo esterno alla famiglia. In misura minore l'episodio della sottrazione avviene nella famiglia con bambini in età scolare $\left(1^{\circ}=32 \% ; 2^{\circ}=35.5 \% ; 3^{\circ}=34 \%\right)$; scarsa invece la fase della famiglia con figli preadolescenti ed adolescenti $\left(1^{\circ}=5 \% ; 2^{\circ}=9.7 \% ; 3^{\circ}\right.$ $=18 \%$ ). Questo dato fa ipotizzare ai Ricercatori che sia più facile per il genitore sottrarre il figlio piccolo, perché crea meno problemi: ad esempio, è più semplice che convincere un adolescente, che ha una capacità cognitiva maggiore di anticipare gli effetti della sottrazione, oltre ad essere anche più in grado di capire in quale situazione familiare si trova, nonché avere altre figure di riferimento oltre alla famiglia.

Diversamente dall'età, per il genere dei bambini sottratti, non emergono differenze importanti tra maschi e femmine: i bambini $\left(1^{\circ}=\right.$ $53.5 \%, 2^{\circ}=45.2 \%, 3^{\circ}=52 \%$ ) vengono sottratti in maniera analoga alle

stato possibile, purtroppo, un confronto sulle caratteristiche personali dei genitori (età, scolarizzazione, lavoro, ecc.); la mancanza di tale dato contestuale ha certamente limitato le ipotesi delle autrici. Un altro dato mancante è rappresentato dalle motivazioni esplicite alla sottrazione da parte dei protagonisti; in questo caso tuttavia, pur in mancanza di tale dato, non si può trascurare quanto il campione sia rappresentato da coppie conflittuali che non sono riuscite ad integrare le differenze al loro interno.

${ }^{6}$ Per conoscere gli Stati che hanno attualmente aderito alla Convenzione si veda: www.hcch.net. Il contributo fa riferimento alla sottrazione avvenuta nei Paesi firmatari che hanno aderito alla Convenzione o almeno in uno dei due Paesi dei partner della coppia mista; nei Paesi non firmatari invece non è possibile conoscere il fenomeno, che è sicuramente più grave data l'assenza di accordi internazionali tra le legislazioni dei Paesi. 
bambine $\left(1^{\circ}=46.5 \% ; 2^{\circ}=54.8 \% ; 3^{\circ}=48 \%\right)$. Sembra, quindi, che l'età sia più importante del genere del figlio.

Il tipo di affidamento presente al momento della sottrazione risulta in evidente maggioranza congiunto $(76 \%)$, in minoranza esclusivo al padre $(10 \%)$ o alla madre $(6 \%)$; nei restanti casi non viene specificato nei dati $(8 \%)$. Anche questo dato diverge dalle precedenti rilevazioni, in cui la possibilità dell'affido congiunto era scarsa $\left(1^{\circ}=10.5 \% ; 2^{\circ}=6.5 \%\right)$, mentre l'affidamento esclusivo alla madre era prevalente $\left(1^{\circ}=\right.$ più dell ${ }^{\prime} 80 \% ; 2^{\circ}=29 \%$ alla madre e $16 \%$ al padre).

Una categoria interessante, ancora in controtendenza rispetto alle precedenti rilevazioni $\left(1^{\circ}, 2^{\circ}\right)$, è rappresentata dal genitore sottraente: al contrario di quello che emergeva dai dati nelle precedenti rilevazioni, è prevalentemente la madre il genitore sottraente $(76 \%)$, mentre si tratta del padre solo nel $22 \%$ dei casi e nei restanti (2\%) sono altri ad essere responsabili della sottrazione, come i nonni materni o paterni (al contrario in $1^{\circ}: 67 \%$ il padre e $33 \%$ la madre; in $2^{\circ}: 61.4 \%$ il padre e $32.3 \%$ la madre, mentre nel $6.4 \%$ altri).

Nuovamente, al contrario di ciò che è emerso nelle precedenti rilevazioni, emergono informazioni significative rispetto alla durata del procedimento, che risulta concluso nella grande maggioranza dei casi $(80 \%)$ entro 6 mesi o entro un anno (12\%); solo nell' $8 \%$ dei casi in un periodo di tempo superiore ad un anno. Diversamente, in $1^{\circ}$ la grande maggioranza dei procedimenti $(86.6 \%)$ ha una durata superiore a un anno e, tra questi, alcuni perdurano fino a 2 anni $(30 \%)$. Solo i pochi rimanenti sono chiusi entro un anno (13.3\%). Ed ancora, in $2^{\circ}$ nell' $82.3 \%$ dei casi il procedimento dura più di un anno, mentre gli altri si risolvono entro l'anno o addirittura in 6 mesi (17.7\%).

Un ultimo dato, altrettanto importante e che non è emerso nei fascicoli delle precedenti rilevazioni, riguarda il rimpatrio del minore, che in questo caso viene disposto nella maggioranza dei casi (68\%). Probabilmente, questo dato si lega all'applicazione della Convenzione e del suo esplicito riferimento al rimpatrio immediato del bambino nel luogo di residenza.

Dai risultati emerge, inoltre, una mancanza di interventi psicologici in tutte e tre le rilevazioni. In particolare, in questa rilevazione effettuata all'Autorità Centrale, gli interventi non sono stati nemmeno considerati: probabilmente l'applicazione sul piano normativo della Convenzione dell'Aja, solleva il Giudice dal disporre interventi di altro tipo, come se si potesse "obbligare" ad un funzionale svolgimento del ruolo 
genitoriale durante la sottrazione. È questo il dato che emerge dalle rilevazioni, ossia che l'applicazione della Convenzione da parte dei Paesi firmatari, ha sollevato il Giudice dal disporre altri interventi di tipo più psicologico: solo nella prima rilevazione, infatti, quando ancora non era stata ratificata la Convenzione, l'intervento era minimamente presente ${ }^{7}$.

\section{Discussione}

L'obiettivo principale che ha guidato il lavoro di questa indagine, è stato di conoscere le caratteristiche del fenomeno della sottrazione internazionale del minore presso l'Autorità Centrale, indicato nella Convenzione dell'Aja come l'Ufficio protagonista di queste situazioni, al fine di contribuire alla necessaria visibilità del fenomeno e di proporre interventi specifici in queste situazioni. La diffusione dei risultati della ricerca dovrebbe rispondere a quest'obiettivo. Innanzitutto, dai dati registriamo un aumento rispetto all'adesione alla Convenzione nel passare degli anni, segno questo che tali accordi internazionali sono considerati importanti dai diversi Paesi nel disciplinare i rapporti legislativi intercorrenti. Questo contribuisce ad un ritorno più sollecito dei bambini sottrattí nei Paesi firmatari, come la Convenzione stabilisce.

I risultati suggeriscono delle differenze rispetto all'indagine preliminare $\left(1^{\circ}\right)$ condotta presso il SSI, prima della ratifica italiana della Convenzione (Tafà et al., 1998), mentre si avvicinano maggiormente alla seconda, condotta successivamente ad essa ( $2^{\circ}$; Tafà, 2009): in altre parole, le rilevazioni condotte al SSI $\left(1^{\circ}\right.$ e $\left.2^{\circ}\right)$ e all'Autorità Centrale $\left(3^{\circ}\right)$, entrambe successive alla ratifica italiana della Convenzione, concordano dunque nei risultati. In entrambe le rilevazioni emerge che, in generale, per quanto riguarda i cambiamenti verificatesi dopo la ratifica, i dati depongono senza alcun dubbio per un successo della Convenzione nel contesto italiano sia in termini di durata del procedimento sia rispetto al rimpatrio del minore, garantito nella grande

\footnotetext{
${ }^{7}$ A questo proposito un contatto informale con le operatrici dell'Autorità Centrale, ha confermato il dato relativo alla mancanza degli interventi psicologici, come emerge dalle indagini, per cui solamente «sono i genitori a farsene carico» in quanto non previsti dalla Convenzione e quindi contro la legge e comunque sarebbero considerati come «un'invadenza alle procedure effettuate dell'altro Stato».
} 
maggioranza dei casi. In tal senso va certamente apprezzato l'apporto della Convenzione, come peraltro i Ricercatori si aspettavano, ossia che l'intervento giuridico è maggiormente evidente relativamente al fenomeno rispetto a quello psicologico. In particolare, l'applicazione della normativa spinge i giudici a stabilire il rimpatrio del bambino e non "premia", dunque, il genitore sottraente conservando la situazione che si è creata con la sottrazione, come emergeva nella rilevazione prima della ratifica $\left(1^{\circ}\right)$, in cui il rapimento del bambino diventava così una sottrazione legittimata (legal kidnapping; Tafà et al., 1998). La decisione del Giudice non è pertanto orientata a conservare lo status quo creatosi con la sottrazione, modificando difficilmente la situazione, anzi sembra voler ripristinare il contesto relazionale in cui ha vissuto il bambino prima del suo rapimento: nel 68\% dei casi, infatti, il minore viene rimpatriato. Questo depone, grazie alla ratifica che velocizza $i$ procedimenti, per un maggior rispetto dei bisogni evolutivi del bambino relativamente allo sradicamento subito in caso di sottrazione dai suoi contesti significativi di appartenenza. Questi dati fanno ipotizzare un'influenza significativa della normativa sulle procedure ed emerge, inoltre, come l'Ufficio dell'Autorità Centrale disposto dalla Convenzione, sembri adempiere ai suoi compiti. La velocizzazione dei procedimenti riscontrata all'interno dél nostro contesto nazionale pare, invece, non trovare conferma nella realtà statunitense, laddove le applicazioni della Convenzione sono delegate sia alle Corti Nazionali (State Courts) che alle Corti Federali (Federal Courts), con probabili resistenze che tale dualismo implica (Lesh, 2011). Le ripercussioni di un siffatto sistema giuridico, peraltro, emergono con maggiore evidenza nei casi di violenza domestica, che caratterizza talvolta l'evento della sottrazione, come alcuni studi riportano (Edleson et al., 2010; Vesneski et al., 2011).

Tornando al contesto italiano e alla rilevazione di questa indagine, un altro dato diverso rispetto alla rilevazione precedente è rappresentato dal "genitore sottraente", che risulta essere in prevalenza la madre: si può ipotizzare che una ragione sia rintracciabile nei cambiamenti legislativi nazionali, che precedentemente garantivano alla madre un affidamento di tipo esclusivo nella grande maggioranza dei casi. A conferma di ciò, in questa rilevazione, come già detto, l'affidamento disposto dal Giudice è per la maggior parte congiunto e non alla madre, come invece accadeva in precedenza (Tafà et al., 1998): questo dato sembra connesso al cambiamento socio-culturale che riguarda il nostro 
Paese, dove l'introduzione dell'affidamento condiviso (Legge $\mathrm{n}^{\circ} 54$ del 2006), ha avviato un cambiamento significativo rispetto al delegare alla madre il ruolo principale nella cura dei figli, a favore di un maggiore coinvolgimento di entrambi i genitori nelle funzioni di accudimento. Probabilmente, questo cambiamento fa sperimentare alla madre l'incertezza del pressoché "esclusivo" affidamento, che invece caratterizzava la situazione precedente ${ }^{8}$.

Si registra un altro elemento di discontinuità nei dati: mentre nella prima rilevazione $\left(1^{\circ}\right)$ la maggior parte dei procedimenti si chiudeva in un tempo superiore ai 2 anni (56.7\%) oppure entro un anno (30\%), successivamente all'applicazione della Convenzione $\left(2^{\circ}\right)$, i procedimenti si concludono in minor tempo ossia in più della metà dei casi $(51.7 \%$ ) entro un anno e nel $20.7 \%$ addirittura entro 6 mesi (Tafà, 2009). In questa terza rilevazione, la durata è ancora inferiore perché nella grande maggioranza dei casi $(80 \%)$, il procedimento di sottrazione si chiude in 6 mesi. Ma anche questo dato, risulta in controtendenza, rispetto allo stesso contesto americano (AA.VV., 2012; Edleson et al., 2010).

La fase del ciclo vitale, che risulta maggiormente a rischio di sottrazione coincide con quella in cui il bambino sottratto è piccolo: in tutte le rilevazioni effettuate, infatti, l'età prescolare risulta essere quella più rappresentata. In accordo con altri studi (Agopian, 1984; Finkelhor, Hotaling, \& Sedlak, 1992; Forehand, Long, \& Zogg, 1989; Greif \& Hegar, 1994), non è, quindi, tanto il genere del bambino, quanto la sua età ad essere importante nella sottrazione (Bordreaux, Lord, \& Stephen, 2000). Conseguentemente con quanto emerso in questi studi, così come dalla nostra indagine, si possono azzardare due ipotesi: la prima riguarda la breve durata di queste unioni miste; la seconda, la maggiore facilità di sottrarre un bambino piccolo, ovvero una facile "preda", rispetto ad un figlio più grande, preadolescente o adolescente.

Rispetto al tipo di affidamento presente al momento della sottrazione, in questa rilevazione prevale quello congiunto rispetto a quello esclusivo: questo dato colpisce rispetto all'esito conflittuale del rapporto di coppia, ossia la sottrazione del bambino. Tale tipo di affidamento, che è il risultato di un accordo tra $\mathrm{i}$ genitori, presuppone una concordanza di visioni educative e non sembra pertanto coerente con quello che accade con l'agito successivo, ossia la sottrazione.

\footnotetext{
${ }^{8}$ Questa ipotesi generale può essere valida solo per le mamme italiane e non per tutto il campione osservato.
} 
Sebbene la procedura sia resa più veloce dall'applicazione della Convenzione ed aumenti le possibilità di rimpatrio per il bambino, tuttavia permane il grosso rischio evolutivo che egli, "s-oggetto" della sottrazione, corre. Colpisce come in una situazione in cui le implicazioni a carico dei protagonisti sono molteplici, la ricerca suggerisca come l'unico intervento attualmente previsto nel contesto nazionale sia quello giuridico. $\grave{E}$ evidente, invece, che gli aspetti giuridici e psicologici vengono ad intrecciarsi. In questo campione, rappresentato ampiamente da bambini in età prescolare e, quindi, con esigui strumenti per far fronte ad una situazione di rischio evolutivo, che non hanno fatto ancora il loro ingresso alla scuola elementare e per i quali la famiglia rappresenta il riferimento relazionale privilegiato nonché l'unico, non è previsto alcun intervento psicologicô sul minore, neanche di sostegno ai genitori, così da supportare le relazioni familiari in queste difficili vicende ${ }^{9}$ (Malagoli Togliatti \& Tafà, 2005; Malagoli Togliatti, Lubrano Lavadera, \& Tafà, 2011; Tafà, 2009; Tafà \& Iesu, 2007). In mancanza di interventi di tipo psicologico, ci si trova dinnanzi all'impotenza di riparare ai danni che la sottrazione ha recato alle relazioni e diviene difficile osservare, valutare, sostenere la relazione genitori-figli (Malagoli Togliatti \& Mazzoni, 2006). Diventa, quindi, arduo tentare di ricostruire la triade, garante della continuità relazionale del bambino con l'esperienza precedente la separazione; con la sottrazione si rischia una vera rottura di questa, poiché non c'è la possibilità di intervenire efficacemente su di essa ${ }^{10}$. Sarebbe invece necessario prevedere un intervento psicologico specifico per queste situazioni familiari, che implicano una brusca rottura delle relazioni nonché numerosi cambiamenti per il minore, fenomeno peraltro in aumento, a causa delle crescenti unioni tra partner di nazionalità diversa nel nostro Paese: quella italiana è ormai indubitabilmente una società multiculturale. Quando, infatti, nei fascicoli esaminati in questa indagine vengono segnalate le ragioni della separazione, esse riguardano prevalentemente la diversità culturale tra i partner. Prendendo le distanze da una visione statistica, è ragionevole ritenere che la sottrazione sia fortemente legata alla profonda diversità di vedute presente nella coppia mista. Il fallimento del lento processo di adattamento reciproco, necessario a tutte le coppie e, in particolare, a

\footnotetext{
${ }^{9}$ Per uno studio rivolto all'analisi del funzionamento del bambino e dei genitori durante la sottrazione si veda il contributo di Forehand, Long, Zogg, \& Parrish, 1989.

${ }^{10} \mathrm{Da}$ qui il titolo di questo contributo. 
quelle miste, a causa delle molteplici diversità - anche di tipo culturale - esistenti tra i partner, potrebbe condurre queste coppie alla contesa dei figli e, quindi, alla loro sottrazione. In particolare, da una ricerca, che ha voluto delineare i profili dei genitori a rischio di sottrazione, emergono il forte legame con il proprio paese di orgine e l'insofferenza al nostro sistema legale (Johnston \& Girdner, 1998).

Relativamente all'assenza degli interventi, nello scenario culturale americano viene realizzata un'azione specifica, anche se limitata solo al momento della riunione del bambino con il genitore che ha subito la sottrazione (Berman-Lippert \& Hatcher, 2009): si tratta, tuttavia, di una possibilità che rimanda $i$ protagonisti ad altri contesti per una richiesta precisamente psicologica.

Il minore, quindi, subisce la trasformazione di iniziali relazioni, che nascono triadiche, in relazioni diadiche: deve scegliere una stirpe cui appartenere, rinunciare non solo ad un genitore, ma anche ai nonni, ai cugini, ad eventuali fratelli. Successivamente al suo rimpatrio, previsto dalla Convenzione, deve velocemente "dimenticare" anche questa nuova situazione relazionale, faticosamente creata con il genitore sottraente, per potersi ricostruire - da solo, senza nessun aiuto - il rapporto con l'altro genitore: il bambino così non può accedere ad entrambe le stirpi, è costretto a scegliere un solo ramo dell'albero vitale che nutre la sua linfa identitaria. In quest'ottica, la sottrazione internazionale del minore può causare la rottura del triangolo primario (Fivaz-Depeursinge \& Corboz-Warnery, 2000), in quanto non si verifica, come può avvenire nella separazione nazionale, semplicemente una riduzione quantitativa della frequentazione genitore/figlio, ma una vera e propria rottura nella relazione, da riparare necessariamente mediante interventi di sostegno alla genitorialità specifici. La soluzione extragiudiziale è la strada meno traumatica per il minore e per i genitori, ed è il percorso da suggerire ai protagonisti, quando la sottrazione è avvenuta. $\mathrm{Ma}$ - come già detto - dall'indagine e dai colloqui informali intrattenuti con gli operatori, non emerge nessun intervento psicologico in queste situazioni ${ }^{11}$ : la richiesta di aiuto psicologico da parte della

\footnotetext{
${ }^{11}$ A questa "distrazione" sfugge l'opuscolo "Bambini contesi - guida per i genitori" (2009) giunto alla sua settima edizione che si rivolge, oltre che ai genitori a cui fornisce dei suggerimenti utili soprattutto dal punto di vista giuridico in queste situazioni, anche ai funzionari della Pubblica Amministrazione chiamati a diverso titolo ad intervenire. L'opuscolo, edito dal Ministero degli Affari Esteri, intende fornire un aiuto alle famiglie che vivono questa situazione di disagio.
} 
famiglia interculturale dipenderebbe, quindi, esclusivamente dall'iniziativa del genitore che ha subito la sottrazione, dalla sua consapevolezza circa la possibilità di un supporto efficace per sé e per il figlio, capace di imporsi sia sulla rabbia e sulla sofferenza per il torto subito, sia sul rimpatrio del figlio con il suo successivo riavvicinamento, che potrebbe rappresentare la soluzione apparente al problema della sottrazione subìta. Non appare allora semplice la possibilità di emergere della richiesta di aiuto da parte della famiglia, mentre sarebbe auspicabile che ci fosse nel momento del rimpatrio del bambino, anziché a causa dei suoi sintomi tardivi. «L'abuso psicologico impedisce al bambino il riconoscimento dei propri bisogni se non attraverso un'espressione sintomatica» (Monaco, Marinucci, \& Viola, 2000; p. 41).

È chiara, quindi, l'importanza di ipotizzare interventi psicologici specifici per le coppie miste. È anche a causa della mancanza di questi (Malagoli Togliatti, Lubrano Lavadera, \& Tafà, 2011), che nella sottrazione si rischia la rottura del triangolo familiare. La sottrazione, infatti, appare come un agito, un acting out, che prende le distanze da una riflessione dei genitori, capace di far valutare la situazione anche rispetto ai figli; è ipotizzabile, quindi, che il bambino si accorga sin da piccolo $^{12}$ che non esiste più una triade, ma diadi separate in conflitto tra di loro, in cui il suo sradicamento, che implica come presupposto la rottura del patto genitoriale, è l'unica modalità di rapporto possibile. La mancanza di interventi di supporto specifici in concomitanza della sottrazione, che rappresenta un segno evidente dell'esasperata conflittualità esistente nella coppia mista separata, si affianca a delle caratteristiche che la letteratura riconduce al rischio di abuso psicologico del minore (Monaco et al., 2000), poiché viene messa a rischio la sua "nicchia ecologica" - intendendo il bambino ed il suo ambiente che si influenzano reciprocamente. In generale, infatti, si tratta di una modalità che non considera e non rispetta i suoi bisogni evolutivi, poiché in ogni caso il bambino è costretto a rinunciare alle proprie esigenze di crescita e a una libera espressione di sé stesso, per adeguarsi alle richieste del genitore sottraente, pena la perdita di un legame affettivo importante, che in quel momento è l'unico percepito esistente nella vita del bambino. Ancora, la sottrazione mette a rischio altri aspetti della vita del bambino:

\footnotetext{
${ }^{12}$ Questo dato, relativo alla piccola età dei bambini sottratti, emerge in tutte e tre le rilevazioni effettuate.
} 
- per esempio, il suo protrarsi nel tempo: infatti, i bambini piccoli subiscono allontanamenti di lunga durata da un genitore, dalla propria stirpe e dal contesto relazionale, socio-culturale di appartenenza - anche se in misura inferiore rispetto agli anni precedenti, grazie alla Convenzione;

- il fatto di subìre l'evento, anzichè essere in un ruolo attivo di protagonista, come emerge dalle rilevazioni effettuate ${ }^{13}$;

- non ultimi in ordine di importanza, i racconti che il genitore sottraente fa dell'altro al figlio, per giustificare il proprio rapimento e spesso, dai racconti informali degli operatori, viene detto ai bambini addirittura che il genitore è morto oppure nella migliore delle ipotesi, lo si descrive in maniera negativa.

Insomma, la sottrazione "distorce" la relazione del bambino con il genitore che ha subito la sottrazione. In queste situazioni, gli strumenti psicologici abitualmente utilizzati nelle separazioni nazionali non vengono applicati: non è possibile effettuare una Mediazione Familiare per dirimere il conflitto nella coppia e per supportare, nonché incoraggiare, i coniugi in una serie di ipotesi sul futuro, a causa dell'ovvia distanza tra le abitazioni dei partner; né tantomeno può essere effettuata una Consulenza Tecnica di Ufficio per valutare le relazioni familiari, in grado di suggerire successivamente l'intervento più adeguato (Malagoli Togliatti \& Mazzoni, 2006). L'incontro con questa famiglia separata diventa molto difficile anche solo per un colloquio preliminare di conoscenza (Tafà, 2007), data la stessa distanza chilometrica. D'altra parte, è necessario affrontare un lutto, quello della coniugalità e dell'unità familiare: eppure in queste situazioni appare come né terapeuti, né mediatori possano gettare le fondamenta che aiutino a fronteggiare le perdite e prevenire quelli che possono essere $\mathrm{i}$ contrasti inevitabili nella coppia. Come afferma Walsh (2008, p. 433): «(...) si affrontano molte perdite con il divorzio. Anche se il partner o il rapporto sono stati profondamente deludenti e dolorosi, il divorzio implica la perdita dell'intera unità familiare e delle speranze e dei sogni che si erano appuntati sul futuro di quelle relazioni». L'aiuto dei terapeuti potrebbe altresì garantire la rivisitazione del rapporto: «(...) $\mathrm{i}$ clinici possono aiutare a ridimensionare i contrasti, ad accrescere un rispetto reciproco tra $\mathrm{i}$ genitori e ad accordarsi su alcune linee guida

\footnotetext{
${ }^{13}$ Nelle precedenti rilevazioni è emerso come la sottrazione non è un evento a cui il bambino viene preparato, ma è per lui imprevisto.
} 
comuni, in modo che ai figli sia garantita una relazione di fiducia e non conflittuale con entrambi i genitori» (Walsh, 2008; p. 432). Questo tipo di legittimazione dell'atto da parte del genitore sottraente, rappresenta un intervento distruttivo della relazione del figlio con l'altro e sembra rimandare a quella campagna di denigrazione rivolta ad un genitore, nota in letteratura come Sindrome di Alienazione Genitoriale (PAS; Gardner, 1985; Gulotta, 1998). Secondo Gardner, si tratta di un disturbo relazionale che presuppone tre protagonisti: il genitore programmatore, che in qualità di alienante, attivamente denigra l'altro ossia il genitore alienato, raccontando eventi che vengono "letti" agli occhi del figlio, come comportamenti di abbandono o tradimento. Il genitore alienante "si serve" pertanto della sottrazione come di un episodio in cui il genitore alienato ha rappresentato, per il figlio, tutt'altro che una figura di sostegno e di protezione. Lo scopo è di separare il figlio dall'altro genitore e di cementarlo a sé: «(...) in questi casi il sentimento di abbandono (e o di tradimento) viene bilanciato da un forte attaccamento verso il genitore che è rimasto» (Malagoli Togliatti \& Franci, 2005; p. 49). Proprio per questo, in precedenza, si è parlato di rischio di abuso psicologico, presente in queste situazioni: a livello clinico, infatti, è forte l'idea di equiparare la Sindrome di Alienazione Genitoriale ad un abuso psicologico vero e proprio, che produce distorsioni evolutive a cui inevitabilmente andrà incontro il bambino (Malagoli Togliatti \& Franci, 2005).

Nonostante i numerosi cambiamenti subiti dal minore con la sottrazione, emerge attualmente la mancanza di interventi specifici sui diversi protagonisti della vicenda familiare:

- la coppia genitoriale in conflitto;

- il bambino sottratto, che subisce l'allontanamento da un genitore e dal suo contesto di appartenenza per vivere con l'altro, di cui è "costretto" ad accettare la "versione della sottrazione"14, dovendosi abituare ad una nuova frequentazione quotidiana, nonché ad un diverso ambiente esterno che implica talvolta l'uso di un'altra lingua. E, quindi, deve mettere da parte la propria individualità a favore di un adattamento ai bisogni degli adulti;

- la relazione intergenerazionale tra il genitore che subìsce la sottrazione ed il figlio, che analogamente subisce una drastica interruzione del rapporto. Come anche la relazione

\footnotetext{
${ }^{14}$ Che - come abbiamo detto - talvolta prevede la morte dell'altro genitore. 55
} 
intergenerazionale tra il bambino ed i nonni, analogamente interrotta dalla sottrazione.

\section{Conclusioni e proposte operative}

Per questo motivo, i risultati di questa indagine possono rappresentare la base per predisporre interventi di politiche sociali specificamente rivolti al sostegno della genitorialità nella sottrazione del minore, che attualmente scarseggiano sul territorio italiano, così come la ricerca suggerisce. A tal proposito, si ritiene che l'intervento offerto dallo Spazio Neutro (Malagoli Togliatti \& Tafà, 2005), che ha come obiettivo quello di ricostruire una relazione funzionale tra le generazioni, sia quello più adeguato a sostenere la genitorialità in queste situazioni caratterizzate da un drastico allontanamento del figlio dallo Stato di residenza abituale. Proprio per l'applicazione della Convenzione dell'Aja, che stabilisce il rientro immediato dei minori nel Paese d'origine, si rivela necessario sostenere e ripristinare una frequentazione interrotta dalla sottrazione, ovviamente nel rispetto dell'interesse del minore. Mettere al centro dell'intervento di sostegno e di quello giudiziario l'interesse del minore, significa che l'intervento va fatto intorno al bambino. L'interesse del minore è, anche in questo tipo di famiglie separate interculturali, quello per cui ogni figlio, separato da uno o entrambi i genitori, deye poter mantenere relazioni personali e contatti diretti in modo regolare con entrambi, salvo quando ciò è decisamente contrario alla sua crescita mentale e relazionale, come suggerisce la Convenzione ONU dei Diritti dell'infanzia (New York, 1989, art. 9), che conferma, in sintonia con la letteratura, il diritto alla bigenitorialità. Lo Spázio Neutro è un luogo ritagliato e libero dal caos distruttivo che il conflitto tra i partner introduce nello scambio tra le diverse generazioni, rappresentate dal genitore e dal figlio. Se un genitore per tanto tempo non ha visto con continuità il figlio e non ha partecipato alla sua educazione, non ci si può aspettare che automaticamente questa relazione riparta senza essere sostenuta. L'accesso ad entrambi i genitori e a entrambe le stirpi, evidenziato dalla letteratura come imprescindibile per un sano sviluppo del bambino, può dunque avvalersi dello Spazio Neutro: luogo d'incontro del bambino con le figure familiari che non ha più visto, sicuramente il genitore, ma anche i nonni. 
Si può ragionevolmente ipotizzare, quindi, che lo Spazio Neutro possa essere inizialmente il modo più adeguato per aiutare il bambino, sostenendo la sua relazione con il genitore che ha subito la sottrazione e da cui torna tramite il rimpatrio. È il genitore che non vede da tempo e con cui deve ripristinare una convivenza bruscamente interrotta, di cui gli è stata raccontata una versione circa il suo ruolo nella sottrazione, talvolta lontana dalla realtà. In questo caso lo Spazio Neutro può rappresentare un contesto di significativo supporto per una relazione che ha subito un allontanamento imprevisto, impreparato e brusco: sia per il bambino, ascoltandolo rispetto all'esperienza sperimentata con il genitore sottraente, a come gli è stata raccontata, a come l'ha vissuta; sia per il genitore, aiutandolo a mettere da parte, il suo risentimento verso il partner, a favore dell'ascolto delle esigenze del figlio. In ogni caso, sarà utile ascoltarlo nei suoi bisogni più profondi. Ciò per enfatizzare l'importanza di un ascolto empatico da parte dell'esperto in queste situazioni, in grado di "entrare in sintonia" con il bambino. Questo conferma, peraltro, l'importanza del "dare voce" al protagonista della vicenda, ossia ascoltare direttamente il minore (Lombardi \& Tafà, 1998; Malagoli Togliatti \& Lubrano Lavadera, 2011). In questo modo, i bambini possono esprimere i propri pensieri ed i propri sentimenti, e trovare finalmente un senso a quello che hanno vissuto, insieme ad un esperto dell'ascolto, che è neutrale, esterno alla famiglia; solo così il bambino può essere assolto dalla responsabilità di tenere insieme $i$ lembi delle differenze culturali espresse dai propri genitori (Mirazita, 2008). La piccola età del bambino sottratto, emersa dalle rilevazioni effettuate nell'indagine, richiede l'uso di tecniche specifiche adatte ai bambini piccoli per riuscire a fare emergere la loro esperienza emotiva, in grado di tener conto del fatto che, talvolta, essi trovano più facile parlare di una situazione "in modo non diretto". È emersa, inoltre, la mancanza di una "preparazione" all'imminente evento della sottrazione da parte del genitore e, quindi, la possibilità di esprimere un dissenso da parte del bambino. Il contesto dell'ascolto è importante anche per dare al bambino una spiegazione chiara degli eventi accaduti, adatta alla sua età, in modo che possa capire, oltre che subìre solamente, quello che gli accade (Malagoli Togliatti \& Lubrano Lavadera, 2011) ${ }^{15}$. Tutto questo

\footnotetext{
${ }^{15}$ Senza pretese di significatività statistica, l'anima clinica di chi scrive, vuole riportare ciò che nei colloqui informali gli assistenti sociali del SSI hanno detto a proposito dell'opportunità di ascoltare il minore in queste situazioni che lo travolgono per conoscere il suo vissuto: «Ma così lui sceglie!» ha replicato un assistente sociale di fronte 57
}

Maltrattamento e abuso all 'infanzia, Vol. 15, n. 2, giugno 2013, pp. 35-64 
sarà possibile se prima l'esperto, in colloqui con i genitori, effettuati anche congiuntamente (se la situazione lo consente), riesce a far capire l'esperienza emotiva del bambino, mettendo da parte quello che ha scatenato la loro conflittualità: il bambino diventa così, per entrambi, la prima cosa a cui pensare. Il lavoro clinico con i genitori, precedente sia al rientro a casa del bambino dal genitore che ha subito la sottrazione, sia all'incontro sempre del bambino con il genitore sottraente prima che lo possa rivedere, potrebbe così tendere ad una possibile ri-narrazione dell'evento, "alla portata" del bambino. Perciò lo Spazio Neutro può essere di aiuto al bambino stesso, per incontrare anche il genitore che lo ha sottratto: quest'intervento può servirgli a reintegrare quella "immagine interna" del genitore che si è "macchiato" della sottrazione, che spesso ha giustificato, mentendo, con la morte dell'altro.

In ogni caso, alto è il rischio che corre la triade familiare in queste situazioni, caratterizzate da un misconoscimento dell'interesse del minore, da un'alta conflittualità tra i genitori non opportunamente trattata attraverso interventi psicologici, resi ulteriormente complessi dal fatto che non possono contare sulla presenza di entrambe le figure genitoriali, indubbiamente necessaria, per la sua riparazione ${ }^{16}$. L'insidia dell'abuso psicologico, che insegue il bambino, costituisce l'esito principale di questo (Faulkner, 1999). Risulta così gravemente compromessa quella che la letteratura definisce la cogenitorialità, ossia la qualità della coordinazione tra gli adulti nei loro ruoli genitoriali (McHale, 2010) e la capacità di supportarsi a vicenda come leader della famiglia (Katz \& Low, 2004).

Un'ipotesi di lavoro ulteriore, oltre allo Spazio Neutro, per salvaguardare il rapporto intergenerazionale, nonché l'ascolto del minore, che aumenterebbe la conoscenza del suo vissuto e delle sue esigenze evolutive, potrebbe essere allora la Mediazione Internazionale, specificamente rivolta alla coppia genitoriale (Montanari, 2007). Si tratta di un intervento effettuato in Francia che, pur originando in

all'opportunità dell'ascolto del minore sollevata dai Ricercatori. Egli voleva così sottolineare come l'ascolto del minore avrebbe certamente reso più difficile la risoluzione del caso di cui era protagonista e si può ipotizzare che questa sia una delle ragioni per cui viene poco realizzato nella pratica. E dai racconti informali degli operatori è emerso come spesso al bambino viene raccontata una versione che talvolta si discosta dalle verità e prevede la morte dell'altro genitore per giustificare la sottrazione.

${ }^{16}$ Di frequente, dopo il fallimento dell'unione di coppia che esita nella separazione e la tentata sottrazione del bambino che si risolve nel suo rimpatrio, il genitore straniero torna a vivere stabilmente nel suo Paese. 
ambito giuridico, attualmente si avvale della collaborazione dei Mediatori dell'Associazione "Ecole des Parents et des Educateurs"17. Si tratta di Mediazioni di breve durata, che tengono conto della distanza abitativa tra i due genitori. Se i genitori si servono efficacemente del contesto di Mediazione Internazionale, potrebbero successivamente accedere allo Spazio Neutro in maniera analogamente proficua. Questo è certamente valido per il genitore e anche per il figlio, che hanno subito la sottrazione, che si accingono a riavviare la convivenza dopo il rimpatrio: infatti, le problematiche psicologiche non sempre terminano con il rientro del bambino. A tal proposito, uno studio (Forehand, Long, \& Zogg, 1989), che ha valutato il funzionamento psicologico dei bambini e dei genitori, che hanno subito la sottrazione, suggerisce che mentre le difficoltà dei genitori erano più evidenti durante la sottrazione, $\mathrm{i}$ bambini invece mostravano più disturbi dopo il rientro $\mathrm{a}$ casa. Inoltre, la stessa Mediazione potrebbe rivelarsi utile anche per l'altro genitore che ne è responsabile, che nel contesto di Mediazione Internazionale potrebbe essere aiutato dall'esperto a distinguere la conflittualità con l'ex partner dal conseguente abuso psicologico perpetuato al figlio con la sottrazione.

In ogni caso, come è evidente, quelle segnate dalla sottrazione rappresentano situazioni molto complesse che vanno valutate da un occhio esperto e vigile, capace di cogliere la situazione nella sua interezza: dal disagio emotivo del bambino al conflitto tra i genitori. Se, da una parte, risulta difficile tracciare traiettorie di intervento comuni a tutte le situazioni caratterizzate dalla sottrazione, dall'altra esse possono aiutare il lavoro degli esperti: sicuramente lo Spazio Neutro può supportare la relazione intergenerazionale; l'ascolto del minore può ampliare la conoscênza della versione soggettiva del bambino, aumentando if vissuto di partecipazione, contro quello di vittima. L'azione di Mediazione Internazionale, inoltre, fornisce all'esperto la possibilità di non dimenticare la triade: il lavoro su di essa, certamente arduo, sia per il conflitto presente nella coppia sia per la distanza chilometrica tra i genitori, rappresenta in ogni caso una preziosa, nonché auspicabile, opportunità per il bambino. Dai risultati che emergono dalle indagini sembra, invece, che ai genitori venga chiesto di arrangiarsi da soli in questa faticosa situazione, e così ai figli di affrontare in solitudine un compito così arduo: al contrario, si rivela necessaria

\footnotetext{
${ }^{17}$ Fondata a Parigi nel 1996 e presente in varie città francesi.
} 59 
un'azione professionale in grado di rammentare agli ex partner il modo migliore di essere genitori e, quindi, contrastare gli effetti traumatici sui bambini. Questo contributo intende sottolineare proprio questa necessità.

\section{Bibliografia}

A.A.V.V. (2012). Litigating international child abduction cases under the hague convention. Alexandria (VA): National Center for Missing \& Exploited Children.

Agopian, M. W. (1984). The impact on children of abduction by parents. Child Welfare, 63, 511-519.

Behrman-Lippert, J., \& Hatcher, C. (2009). Psychological issues in recovery and family reunification. In National Center for Missing \& Exploited Children (Eds.), Family abduction. prevention and response (disponibile online: http://www.missingkids.com/en_US/publications/NC75.pdf).

Bordreaux, M. C., Lord, W. D., \& Stephen, E. E. (2000). Child abduction: An overview of current and historical perspectives. Child Maltreatment, 5, 1, 63-71, doi: 10.1177/1077559500005001008.

Burk, C. (2004). Living in several languages: Implications for therapy. Journal of Family Therapy, 26, 4, 314-339, doi: 10.1111/j.1467-6427.2004.00287.x.

Carli, L., \& Rodini, C. (2008). Le forme dell'intersoggettività. Milano: Cortina.

Chan, A. Y., \& Wethington, E. (1998). Factors promoting marital resilience among interracial couples. In H. I. McCubbin (Ed.), Resiliency in native American and immigrant families (pp. 71-87). Thousand Oaks: Sage.

Cigoli, V. (1998). Psicologia della separazione e del divorzio. Milano: FrancoAngeli.

Cigoli, V. (2006). L'albero della discendenza. Clinica dei corpi familiari. Milano: FrancoAngeli.

Douzenis, A., Kontoangelos, K., Thomadaki, O., Papadimitriou, G. N., \& Lykouras, E. (2012). Abduction of children by their parents: A psychopathological approach to the problem in mixed-ethnicity marriages. British Journal of Medicine \& Medical Research, 2, 3, 405-412.

Dowling, E., \& Gorell Barnes, G. (2000). Working with children and parents through separation and divorce. Basingstoke: Palgrave Macmillan. Tr. it. (2004), Lavorare con i bambini e i genitori nel processo di separazione e divorzio. Milano: FrancoAngeli.

Edleson, J. L., Lindhorst, T., Mehrotra, G., Vesneski, W., Lopez, L., \& Shetty, S. (2010). Multiple perspectives on battered mothers and their children fleeing to the United States for Safety: A study of hague Convention cases. Washington, DC: US Department of Justice, National Institute of Justice. 
Emery, R. E. (2004). The truth about children and divorce. Dealing with the emotions so you and your children can thrive. London: Cambridge. Tr. it. (2008), La verità sui figli e il divorzio. Milano: FrancoAngeli.

Faulkner, N. (1999). Parental child abduction is child abuse. Presented to the United Nations Convention on Child Rights in Special Session, June 9, 1999 (disponibile online: www. prevent-abuse-now.com).

Favaro, G. (1996). Da radici diverse. Famiglia mista e scelte educative. In M. Tognetti Bordogna (Ed.), Legami familiari e immigrazione: $i$ matrimoni misti. Torino: L'Harmattan.

Finkelhor, D., Hotaling, G. T., \& Sedlak, A. J. (1992). The Abduction of Children by Strangers and Nonfamily Members: Estimating the Incidence Using Multiple Methods. Journal of Interpersonal Violence, 7, 2, 226-243, doi: 10.1177/088626092007002008.

Fivaz-Depeursinge, E., \& Corboz-Warnery, A. (1999). The primary triangle. San Francisco: Jossey-Bass. Tr. it. (2000), Il triangolo primario: le prime interazioni triadiche tra padre, madre e bambino. Milano: Cortina.

Forehand, R., Long, N., \& Zogg, C. (1989). Parental child abduction: The problem and possible solution. In B. B. Lahey \& A. E. Kazdin (Eds.), Advances in clinical child psychology (Vol. 12) (pp. 113-177). New York: Plenum.

Forehand, N., Long, N., Zogg, C., \& Parrish, E. (1989). Parent child functioning following return. Clinical Pediatrics, 28, 7, 311-316, doi: 10.1177/000992288902800703.

Gambini, P. (2007). Psicologia della famiglia. La prospettiva sistemicorelazionale. Milano: FrancoAngeli.

Gardner, R. A. (1985). Recent trends in divorce and custody litigation. The Academy Forum, 29, 2, 3-7.

Gollini, A., Tafà, M., \& Malagoli Togliatti, M. (2001). Gli adolescenti e la qualità della comunicazione nelle famiglie separate. Rivista di Sessuologia Clinica, 2, 21-38.

Gozzoli, C., \& Regalia, C. (2005). Famiglie e migrazione. Bologna: il Mulino.

Gregori, A. (1998). La sottrazione internazionale dei minori figli di coppie miste separate. Quaderni per l'infanzia, 4, 54-60.

Greif, L. G., \& Hegar R. L., (1994). Parents who abduct: A qualitative study with implications for practice. Family Relations, 43, 3, 283-289.

Gulotta, G. (1998). La sindrome di alienazione genitoriale: definizione e descrizione. Pianeta Infanzia, 4, 27-36.

Hetherington, E. M., Law, T. C., \& O’Connor, T. G. (1993). Divorce: Challenges, changes, and new chanches. In F. Walsh (Ed.), Normal family processes ( $2^{\text {nd }}$ ed.; pp. 208-234). New York: Guildford Press.

Iesu, L., Fiorelli, M., \& Tafà, M. (2008). Quando la coppia mista si separa: la sottrazione internazionale del minore. Sviluppi della ricerca. Poster presentato al Congresso Nazionale della Sezione di Psicologia Clinica e

61

Maltrattamento e abuso all'infanzia, Vol. 15, n. 2, giugno 2013, pp. 35-64 
Dinamica, AIP - Associazione Italiana di Psicologia, (Padova, Cleup, 12-14 settembre).

Johnston, R. L., \& Girdner, L. K. (1998). Early identificatioon of risk factors for parental abduction. Family \& Conciliation Court Review, 36, 1, 32-40.

Katz, F., \& Low, S. M. (2004). Marital divorce, co-parenting, and family-level processes in relation to children's adjustment. Journal of Family Psychology, 18, 372-382.

Lesh, E. (2011). Jurisdiction friction and the frustration of the Hague convention: Why international child abduction cases should be heard exclusively by Federal courts. Family Court Review, 49, 1, 170-189, doi: 10.1111/j.1744-1617.2010.01360.x.

Lombardi, R., \& Tafà, M. (1998). Ascoltare il minore ovvero entrare in relazione. MinoriGiustizia, 4, 78-92.

Malagoli Togliatti, M., \& Franci, M. (2005). La Sindrome di Alienazione Genitoriale (PAS): studi e ricerche. Maltrattamento e abuso all infanzia, 3, 39-62, doi: 10.1400/63077.

Malagoli Togliatti, M., \& Lubrano Lavadera, A. (2008). Sul concetto di cogenitorialità nelle famiglie unite e separate: nodi teorici ed empirici. Età Evolutiva, 2, 99-115.

Malagoli Togliatti, M., \& Lubrano Lavadera, A. (2011). Bambini in tribunale. Milano: Cortina.

Malagoli Togliatti, M., Lubrano Lavadera, A., \& Tafà, M. (2011). Quando la cogenitorialità diventa difficile da realizzare: la sottrazione internazionale del minore. Poster presentato al Congresso Nazionale della Sezione di Psicologia Clinica e Dinamica, AIP (Catania, 16-18 settembre).

Malagoli Togliatti, M., \& Mazzoni, S. (2006). Osservare, valutare sostenere la relazione genitori-figli. Milano: Cortina.

Malagoli Togliatti, M., \& Tafa,, M. (2005). Gli interventi sulla genitorialità nei nuovi Centri per le Famiglie. Esperienze di ricerca. Milano: FrancoAngeli.

McHale, J. P. (2007). Charting the bumpy road of coparenthood: Understanding the challenger of family life. Washinhton: Zero to Three National Center for Infants, Toddlers and Families Press. La sfida della cogenitorialità (Tr. it., 2010). Milano: Raffaello Cortina Editore.

McHale, J. P. (2010). Charting the Bumpty Road of Coparenthood: Understanding the Challenger of Family Life. Zero to Three: National Center for Infants. Toddlers and Families La sfida della cogenitorialità. Milano: Raffaello Cortina Editore.

Montanari, I. (2007). Separazione e genitorialità. Quaderni del Centro Famiglia, 24. Milano: Vita e Pensiero.

Mazzoni, M., \& Tafà, M. (2007). L'intersoggettività nella famiglia. Procedure multi-metodo per l'osservazione e la valutazione delle relazioni familiari. Milano: FrancoAngeli. 
Minuchin, S. (1974). Families and family therapy. Cambridge: Harvard University Press. Tr. it. (1976), Famiglie e terapie della famiglia. Roma: Astrolabio.

Mirazita, P. (2008). La famiglia straniera nel contesto coatto. Le difficoltà nella valutazione e le sfide della presa in carico. Terapia Familiare, 87, 29-50.

Monaco, W., Marinucci, S., \& Viola, S. (2000). Conflittualità genitoriale e rischio di abuso psicologico. Maltrattamento e abuso all'infanzia, 2, 1, 3746, doi: 10.1400/62945.

Pazè, P. (2008). Le garanzie processuali nel procedimento civile per la sottrazione internazionale di minori. La Nuova Giurisprudenza Civile Commentata, 3, 372-387.

Re, P., \& Sanchez, A. M. (2003). La sottrazione internazionale di minori: aspetti psicologici. Diritto di Famiglia e delle Persone, II, 577-588.

Scabini, E., \& Cigoli, V. (2000). Il famigliare. Legami, simboli e transizioni. Milano: Cortina.

Simonelli, A., Bighin, M., \& De Palo, F. (2012). Il Lausanne Trilogue Play. Milano: Cortina.

Tafà, M. (1999). La sottrazione internazionale dei minori: risultati di un'indagine condotta al Servizio Sociale Internazionale di Roma. Intervento orale al IV Congresso Internazionale della Società Italiana di Psicologia e Psicoterapia Relazionale (S.I.P.P.R.), Individui e relazioni intergenerazionali nella famiglia, 674-676.

Tafà, M. (2001a). Il concetto di Sé nei figli dei separati: difficoltà relazionali e percezione di autoefficacia durante 1'adolescenza. Psicologia Clinica dello Sviluppo, 2, 207-222.

Tafà, M. (2001b). The concept of self in children of split parents: relational impairments and perception of self-efficacy in adolescence. Poster presentato al $3^{\circ}$ Congrès Européenne de Psychopathologie de l'Enfant et de l'Adolescent. Psychopathologie et Parentalités (Lisbona, 31 Maggio-2 Giugno)

Tafà, M. (2007). Il colloquio clinico nell'ottica sistemico-relazionale. In M. Mazzoni \& (M.) Tafà (Eds.), L'intersoggettività nella famiglia. Procedure multi-metodo per l'osservazione e la valutazione delle relazioni familiari (pp. 79-93). Milano: FrancoAngeli.

Tafà, M. (2009). Il fenomeno della sottrazione internazionale del minore: risultati di una ricerca. Minorigiustizia, 2, 116-127.

Tafà, M., \& Iesu, L. (2007). Quando la coppia mista si separa: la sottrazione internazionale del minore. Intervento orale al IX Congresso Nazionale della Sezione di Psicologia Clinica e Dinamica, AIP (Perugia, 28-30 settembre).

Tafà, M., Malagoli Togliatti, M., \& Milazzo, B. (1998). La sottrazione internazionale dei minori: un contributo d'indagine. Psicologia Clinica dello Sviluppo, 2, 341-360. 
Tafà, M., \& Micci, A. (2004). A study on communicative dynamics in the family units: Parents separated in comparison with adolescent sons. Intervento orale al $4^{\circ}$ Congrès de l'Association Européenne de Psychopathologie de l'Enfant et de l'Adolescent (Aepea; Parigi, 28-30 ottobre).

Tajfel, H., \& Turner, J. C. (1986). The social identity theory of inter-group behavior. In S. Worchel \& L. W. Austin (Eds.), Psychology of intergroup relations. Chicago: Nelson-Hall.

Usita, P., \& Poulsen, S. (2003). Interracial relationships in Hawaii: Issues, benefits and therapeutic interventions. Journal of Couple and Relationship Therapy, 2, 73-83, doi: 10.1300/J398v02n02_06.

Veneski, W. M., Lindhorst, T., \& Edelson, J. L. (2011). U.S. judicial implementation of the Hague Convention in cases alleging domestic violence. Juvenile \& Family Court Journal, 62, 2, 1-21, doi: 10.1111/j.1755-6988.2011.01058.x.

Walsh, F. (2006). Strengthening family resilience. New York: The Guilford Press. Trad. it. (2008). La resilienza familiare. Milano: Cortina.

Pervenuto settembre 2012

Accettato aprile 2013 